\title{
A CANONICAL DIMENSION ESTIMATE FOR NON-SPLIT SEMISIMPLE $p$-ADIC LIE GROUPS
}

\author{
KONSTANTIN ARDAKOV AND CHRISTIAN JOHANSSON
}

\begin{abstract}
We prove that the canonical dimension of an admissible Banach space or a locally analytic representation of an arbitrary semisimple $p$-adic Lie group is either zero or at least half the dimension of a non-zero coadjoint orbit. This extends the results of Ardakov, Wadsley, and Schmidt in the split semisimple case.
\end{abstract}

\section{INTRODUCTION}

This paper can be regarded as a postscript to AW1. Its purpose is to record an argument that extends [AW1, Theorem A] to compact semisimple $p$-adic analytic groups whose Lie algebra is not necessarily split ( $p$ is a prime number). An analogue of [AW1, Theorem A] for distribution algebras was proved by Schmidt in Sch2, Theorem 9.9]; our argument works to extend his result as well. For simplicity, we mostly focus on the setting of Iwasawa algebras in this introduction. Let us recall that if $G$ is a compact $p$-adic analytic group and $K G$ is its completed group ring (or Iwasawa algebra) with respect to a finite field extension $K / \mathbb{Q}_{p}$, then the category $\mathcal{M}$ of finitely generated $K G$-modules is an abelian category anti-equivalent to the category of admissible $K$-Banach space representations of $G$ ([ST1]). $\mathcal{M}$, and related categories of $p$-adic representations of (locally) compact $p$-adic analytic groups have received a lot of attention in the last few decades, motivated by research in the Langlands programme and Iwasawa theory. Each object $M$ in $\mathcal{M}$ has a nonnegative integer $d(M)=d_{K G}(M)$ attached to it called its canonical dimension. This notion gives rise to a natural filtration of $\mathcal{M}$,

$$
\mathcal{M}=\mathcal{M}_{d} \supseteq \mathcal{M}_{d-1} \supseteq \cdots \supseteq \mathcal{M}_{0},
$$

by Serre subcategories, where $d=\operatorname{dim} G$ and $M \in \mathcal{M}_{i}$ if and only $d(M) \leq i$. The canonical dimension $d(M)$ may be taken as a measure of the "size" of $M$; for example, it is known that $d(M)=0$ if and only $M$ is finite dimensional as a $K$-vector space, and $d(M)<d$ if and only if $M$ is torsion. It may also be regarded as a non-commutative analogue of the dimension of the support of a module in the commutative setting. The following result, together with its analogue for distribution algebras (Theorem 2) is the main result of this note:

Theorem 1. Let $G$ be a compact p-adic analytic group whose Lie algebra $\mathcal{L}(G)$ is semisimple, and let $G_{\mathbb{C}}$ denote a complex semisimple algebraic group with the same root system as $\mathcal{L}(G) \otimes_{\mathbb{Q}_{p}} \overline{\mathbb{Q}}_{p}$. Let $M$ be a finitely generated $K G$-module which is

Received by the editors July 6, 2015 and, in revised form, December 31, 2015.

2010 Mathematics Subject Classification. Primary 11F85, 16S99, 22E50.

Key words and phrases. p-adic Banach space representations, locally analytic representations, canonical dimension. 
infinite dimensional as a $K$-vector space, and assume that $p$ is an odd very good prime for $G$. Then $d_{K G}(M) \geq r$, where $r$ is half the smallest possible dimension of a non-zero coadjoint $G_{\mathbb{C} \text {-orbit. }}$

For the definition of a very good prime see [AW1, §6.8]. We recall that Theorem A of [AW1 proves the same conclusion under the additional hypothesis that $\mathcal{L}(G)$ is split semisimple. Since the Langlands programme often deals with groups that are not split, it seemed natural to want to remove this assumption from the theorem. Examples of such non-split groups are $G=\mathbb{G}(K)$, where $\mathbb{G} / K$ is an anisotropic semisimple algebraic group.

The proof is a variation of that of the split case. Let us first give a short rough description of the split case and refer to the introduction of AW1 for more details. A short argument reduces the problem to the case when $G$ is a uniform pro- $p$ group. This implies we may associate to $G$ a certain $\mathbb{Z}_{p}$-Lie algebra $\mathfrak{h}$. The algebra $K G$ comes with a morphism into an inverse system $\left(D_{w}=D_{p^{-1 / w}}(G, K)\right)_{w=p^{n}}$ of distribution algebras of $G$, whose inverse limit is the locally analytic distribution algebra $D(G, K)$ with coefficients in $K$ studied in detail by Schneider and Teitelbaum. For large enough $w=p^{n}, D_{w} \otimes_{K G} M \neq 0$. The algebra $D_{w}$ turns out to be a crossed product $\mathcal{U}_{n} * G / G^{p^{n}}$, where $\mathcal{U}_{n}=\widehat{U\left(p^{n} \mathfrak{h}\right)_{K}}$ is an affinoid enveloping algebra, and it turns out that it is enough to prove the analogous result for the algebras $\mathcal{U}_{n}$. The proof of this analogue relies on some technical work: analogues of Beilinson-Bernstein localisation, Quillen's Lemma and Bernstein's Inequality. The assumption that $\mathcal{L}(G)$ is split comes up in the localisation theory, which works for affinoid enveloping algebras of $\mathcal{O}_{L}$-Lie algebras that are of the form $\pi^{m} \mathfrak{g}$, with $L / \mathbb{Q}_{p}$ a finite extension, $\pi$ a uniformizer in $L$ and $\mathfrak{g}$ a split Lie algebra over $\mathcal{O}_{L}$.

In this paper we follow the same reduction steps to get non-zero $\mathcal{U}_{n}$-modules $D_{p^{n}} \otimes_{K G} M$ for sufficiently large $n$. Since the techniques of [AW1] are not strong enough to deduce a canonical dimension estimate for the affinoid enveloping algebra $\mathcal{U}_{n}$ in this case, we base change to a finite extension $L / \mathbb{Q}_{p}$ such that the Lie algebra $\mathcal{L}(G) \otimes_{\mathbb{Q}_{p}} L$ is split and hence has a split lattice $\mathfrak{g}$ in addition to the lattices $p^{n} \mathfrak{h} \otimes_{\mathbb{Z}_{p}} \mathcal{O}_{L}$. We then sandwich a $p$-power multiple of $\mathfrak{g}$ in between two sufficiently large $p$-power multiples of $\mathfrak{h} \otimes_{\mathbb{Z}_{p}} \mathcal{O}_{L}$ in order to base change one of the $D_{p^{n}} \otimes_{K G} M$ to an affinoid enveloping algebra for which the theory of [AW1 applies.

As remarked earlier, Schmidt gave an analogue of [AW1, Theorem A] for coadmissible modules of the distribution algebra $D(G, K)$, using the methods of [AW1]. Here we may now allow $G$ to be a locally $L$-analytic group for some intermediate extension $K / L / \mathbb{Q}_{p}$. Our argument works equally well in this setting to remove the splitness hypothesis of [Sch2, Theorem 9.9], giving us the following theorem:

Theorem 2. Let $G$ be a locally L-analytic group whose Lie algebra $\mathcal{L}(G)$ is semisimple, and let $G_{\mathbb{C}}$ denote a complex semisimple algebraic group with the same root system as $\mathcal{L}(G) \otimes_{L} \overline{\mathbb{Q}}_{p}$. Let $M$ be a coadmissible $D(G, K)$-module such that $d_{D(G, K)}(M) \geq 1$ and assume that $p$ is an odd very good prime for $G$. Then $d_{D(G, K)}(M) \geq r$, where $r$ is half the smallest possible dimension of a non-zero coadjoint $G_{\mathbb{C} \text {-orbit. }}$

We remark that Theorem 1 follows from Theorem 2 by the faithful flatness of the distribution algebra over the Iwasawa algebra ([ST2, Theorem 5.2]). Nevertheless, we have opted to give independent arguments, keeping the proof of Theorem 1 independent of [Sch2, Corollary 5.13] (in the case $L=\mathbb{Q}_{p}$ ), which was conjectured 
in AW1 but neither proved nor needed. We also remark that, for a coadmissible $D(G, K)$-module $M, d_{D(G, K)}(M)=0$ if and only if $D_{w} \otimes_{D(G, K)} M$ is a finitedimensional $K$-vector space for all $w$.

Let us now describe the contents of this paper. Section 2 proves the necessary flatness results needed to make the sandwich argument work and recalls some generalities on crossed products. The flatness result is a consequence of a general result of Berthelot and Emerton. Section 3 introduces the main players, discusses some complements to results in AW1, and then proves Theorem 1 fleshing out the strategy described above. Finally, Section 4 proves Theorem 2 .

\section{Flatness And CROSSED PROduCts}

Fix a prime number $p$ and let $K$ be a finite extension of $\mathbb{Q}_{p}$. In this section we give a condition for the natural map between affinoid enveloping algebras induced from an inclusion of finite free $\mathcal{O}_{K}$-Lie algebras of the same rank to be left and right flat. All completions arising in this paper are $p$-adic completions. We use a technique due to Berthelot, abstracted by Emerton in [Em, Proposition 5.3.10].

Lemma 3. Let $\mathfrak{h} \subseteq \mathfrak{g}$ be $\mathcal{O}_{K}$-Lie algebras, both finite free of rank d. Assume that $[\mathfrak{g}, \mathfrak{h}] \subseteq \mathfrak{h}$. Then $\widehat{U(\mathfrak{g})}_{K}$ is a flat left and right $\widehat{U(\mathfrak{h})}_{K}$-module.

Proof. The proof is very close to that of [SS, Proposition 3.4], which contains the case $\mathfrak{h}=p \mathfrak{g}$ as a special case. First we prove right flatness. Put $A=U(\mathfrak{h})$ and $B=U(\mathfrak{g})$ and let $F_{\bullet} A$, respectively, $F_{\bullet} B$ denote the usual Poincaré-BirkhoffWitt filtrations on $A$ and $B$, respectively. Since $A$ and $B$ are free $\mathcal{O}_{K}$-modules by [Bour, $\S I .2 .7$, Corollaire 3], we see that they are $p$-torsion-free and $p$-adically separated left Noetherian $\mathbb{Z}_{p}$-algebras. Define a new increasing filtration on $B$ by the $\mathbb{Z}_{p}$-submodules

$$
F_{i}^{\prime} B=A \cdot F_{i} B=\sum_{j \leq i} A \mathfrak{g}^{j} .
$$

In order to apply [Em, Proposition 5.3.10], we need to verify conditions (i)-(iii) in the statement of [Em, Lemma 5.3.9]. Condition (ii), that $F_{0}^{\prime} B=A$, is clear. Condition (i) asks that $F_{i}^{\prime} B \cdot F_{j}^{\prime} B \subseteq F_{i+j}^{\prime} B$. To see this, note that because $\mathfrak{g}$ normalises $\mathfrak{h}$, we have $[\mathfrak{g}, A] \subseteq A$, so $\mathfrak{g} A \subseteq A \mathfrak{g}+A$. Hence $\mathfrak{g}^{i} A \subseteq F_{i}^{\prime} B$ by an easy induction, so $A \mathfrak{g}^{i} A \mathfrak{g}^{j} \subseteq F_{i+j}^{\prime} B$ as required. Condition (iii) asks that $G r^{F^{\prime}} B$ is finitely generated over $A$ by central elements. Since $F_{\bullet} B \subseteq F_{\bullet}^{\prime} B$, the identity map induces a natural map $f: G r_{\bullet}^{F} B \rightarrow G r_{\bullet}^{F^{\prime}} B$ and since $G r_{0}^{F^{\prime}} B=A$ we see that $G r_{\bullet}^{F^{\prime}} B$ is generated (as a ring) by $f\left(G r_{\bullet}^{F} B\right.$ ) and $A$. Now $G r_{\bullet}^{F} B=\operatorname{Sym}(\mathfrak{g})$, thus we may find $X_{1}, \ldots, X_{d} \in \mathfrak{g}$ whose symbols $g r^{F^{\prime}} X_{k} \in G r_{1}^{F^{\prime}} B$ generate $G r_{\bullet}^{F^{\prime}} B$ over $A$. Thus we have shown finite generation, and to show that the $g r^{F^{\prime}} X_{k}$ are central it suffices to prove that for any $X \in \mathfrak{g}, g r^{F^{\prime}} X$ commutes with $A$ in $G r_{\bullet}^{F^{\prime}} B$. Since $A$ is generated by $\mathfrak{h}$ it suffices to verify this for $Y \in \mathfrak{h} \subseteq G r_{0}^{F^{\prime}} B$. Computing, we see that $\left(g r^{F^{\prime}} X\right)\left(g r F^{F^{\prime}} Y\right)-\left(g r^{F^{\prime}} Y\right)\left(g r^{F^{\prime}} X\right)$ is equal to the image of the bracket $[X, Y]$ inside $G r_{1}^{F^{\prime}} B$. By assumption $[X, Y] \in \mathfrak{h}$, so it becomes 0 in $G r_{1}^{F^{\prime}} B$. Having verified the assumptions of [Em, Proposition 5.3.10] we may conclude that $\widehat{B}_{K}$ is a flat right $\widehat{A}_{K}$-module.

To prove left flatness we recall from [Bour, §I.2.4, Proposition 4] that $U(\mathfrak{h})^{\text {op }} \rightarrow$ $U(\mathfrak{g})^{\text {op }}$ is canonically isomorphic to $U\left(\mathfrak{h}^{\text {op }}\right) \rightarrow U\left(\mathfrak{g}^{\text {op }}\right)$, where we recall that the opposite Lie algebra $\mathfrak{h}^{\text {op }}$ of $\mathfrak{h}$ is the $\mathcal{O}_{K}$-module $\mathfrak{h}$ together with a new bracket $[-,-]^{\prime}$ 
defined by $[X, Y]^{\prime}=[Y, X]$. Thus, having proved right flatness of $U\left(\mathfrak{h}^{\text {op }}\right) \rightarrow U\left(\mathfrak{g}^{\text {op }}\right)$ above we may deduce left flatness of $U(\mathfrak{h}) \rightarrow U(\mathfrak{g})$.

Next let us recall the notion of a crossed product from, e.g., [Pa, §1]. Let $S$ be a ring and let $H$ be a group. A crossed product $S * H$ is a ring containing $S$ as a subring and a subset $\bar{H}=\{\bar{h} \mid h \in H\}$ of units bijective with $H$ that satisfies the following conditions:

- $S * H$ is a free right $S$-module with basis $\bar{H}$.

- We have $\bar{h} S=S \bar{h}$ and $\overline{g h} S=\bar{g} \bar{h} S$ for all $g, h \in H$.

Given a crossed product $S * H$ we obtain functions $\sigma: H \rightarrow \operatorname{Aut}(S)$ and $\tau: H \times H \rightarrow S^{\times}$, called the action and the twisting, defined by

$$
\begin{gathered}
\sigma(h)(s):=(\bar{h})^{-1} s \bar{h}, \\
\tau(g, h)=(\overline{g h})^{-1} \bar{g} \bar{h} .
\end{gathered}
$$

The associativity of $S * H$ is equivalent to certain relations between $\sigma$ and $\tau$ ([Pa, Lemma 1.1]) and conversely, given $S, H, \sigma$ and $\tau$ satisfying these relations we may construct $S * H$ as the free right $S$-module on a set $\bar{H}=\{\bar{h}: h \in H\}$ which is in bijection with $H$, and multiplication being defined by extending the rule

$$
(\bar{g} r)(\bar{h} s)=\overline{g h} \tau(g, h) r^{\sigma(h)} s
$$

additively $(g, h \in H, r, s \in S)$. Here we use the notation $s^{f}$ to denote the (right) action of $f \in \operatorname{Aut}(S)$ on $s \in S$. Let us record how crossed products behave with respect to taking opposites.

Lemma 4. Let $S * H$ be a crossed product of a ring $S$ by a group $H$, with action $\sigma: H \rightarrow \operatorname{Aut}(S)$ and twisting $\tau: H \times H \rightarrow S^{\times}$.

1) $(S * H)^{\mathrm{op}}=S^{\mathrm{op}} * H^{\mathrm{op}}$, with action $\sigma^{\mathrm{op}}$ and twisting $\tau^{\mathrm{op}}$ on the right-hand side given by

$$
\begin{gathered}
\sigma^{\mathrm{op}}(h)=\sigma(h)^{-1}, \\
\tau^{\mathrm{op}}(g, h)=\tau(h, g)^{\sigma(g)^{-1} \sigma(h)^{-1}} .
\end{gathered}
$$

2) Let $T$ be another ring with a homomorphism $\phi: S \rightarrow T$ and let $\Gamma \subseteq \operatorname{Aut}(S)$ be a subgroup of the automorphisms of $S$ that contains the inner automorphisms defined by $\tau(g, h)$ for $g, h \in H$ and the $\sigma(h)$ for $h \in H$. We assume that there is a compatible homomorphism $\psi: \Gamma \rightarrow \operatorname{Aut}(T)$, where by compatible, we mean that for $f \in \Gamma, \phi \circ f=\psi(f) \circ \phi$, and additionally that $\psi$ sends the inner automorphism defined by $\tau(g, h) \in S^{\times}$to the inner automorphism defined by $\phi(\tau(g, h))$ for all $g, h \in H$. Then we may form a crossed product $T * H$ with action $\psi \circ \sigma$ and twisting $\phi \circ \tau$. Moreover, $\phi$ extends naturally to a homomorphism $\Phi: S * H \rightarrow T * H$.

3) The constructions in 1) and 2) are compatible: Given the situation in 2), we also have $\phi: S^{\mathrm{op}} \rightarrow T^{\mathrm{op}}$ and $S^{\mathrm{op}} * H^{\mathrm{op}}$; we may form $T^{\mathrm{op}} * H^{\mathrm{op}}$ and we obtain a natural map $S^{\mathrm{op}} * H^{\mathrm{op}} \rightarrow T^{\mathrm{op}} * H^{\mathrm{op}}$. Under the identification in 1) this agrees with $(T * H)^{\mathrm{op}}$ and $\Phi$.

Proof. 1) $(S * H)^{\mathrm{op}}$ contains $S^{\mathrm{op}}$ as a subring as well as the set $\bar{H}$ which is bijective with $H^{\text {op }}$. To verify that $(S * H)^{\text {op }}$ is a crossed product $S^{\text {op }} * H^{\text {op }}$ it remains to verify that, working in $S * H, S \bar{h}$ is a free left $S$-module and that $S \overline{g h}=(S \bar{g}) \bar{h}$ for all $g, h \in H$. The first assertion follows from $s \bar{h}=\bar{h} s^{\sigma(h)}$ and that $\bar{h} S$ is a free right 
$S$-module and $\sigma(h)$ is an automorphism of $S$. The second follows similarly using the formula

$$
\bar{g} \bar{h}=\tau(g, h)^{\sigma(g h)^{-1}} \overline{g h} .
$$

We may then compute the formulae for $\sigma^{\mathrm{op}}$ and $\tau^{\mathrm{op}}$, using $\cdot$ to distinguish the multiplication in $(S * H)^{\text {op }}$ or $H^{\text {op }}$ from that in $S * H$ or $H$ :

$$
\begin{gathered}
s^{\sigma^{\mathrm{op}}(h)}=(\bar{h})^{-1} \cdot s \cdot \bar{h}=\bar{h} s(\bar{h})^{-1}=s^{\sigma(h)^{-1}}, \\
\tau^{\mathrm{op}}(g, h)=(\overline{g \cdot h})^{-1} \cdot \bar{g} \cdot \bar{h}=\bar{h} \bar{g}(\overline{h g})^{-1}=\tau(h, g)^{\sigma(g)^{-1} \sigma(h)^{-1}} .
\end{gathered}
$$

This finishes the proof of (1). We now prove (2). First, it is straightforward to verify that $\psi \circ \sigma$ and $\phi \circ \tau$ satisfy $[\mathrm{Pa}$, Lemma 1.1] using the compatibility condition. Thus we may form $T * H$ as above, and $\phi$ induces an additive group homomorphism $\Phi: S * H \rightarrow T * H$ given by $\Phi(\bar{h} s)=\bar{h} \phi(s)$ which is easily checked to be a ring homomorphism. Finally, to check 3 ) we note that

$$
(\psi \circ \sigma)^{\mathrm{op}}(h)=\psi(\sigma(h))^{-1}=\psi\left(\sigma(h)^{-1}\right)=\psi\left(\sigma^{\mathrm{op}}(h)\right)
$$

and

$$
\begin{aligned}
(\phi \circ \tau)^{\mathrm{op}}(g, h) & =\phi(\tau(h, g))^{\psi(\sigma(g))^{-1} \psi(\sigma(h))^{-1}}=\phi(\tau(h, g))^{\psi\left(\sigma(g)^{-1} \sigma(h)^{-1}\right)} \\
& =\phi\left(\tau(h, g)^{\sigma(g)^{-1} \sigma(h)^{-1}}\right)=\phi\left(\tau^{\mathrm{op}}(g, h)\right) .
\end{aligned}
$$

This shows that $(T * H)^{\mathrm{op}}=T^{\mathrm{op}} * H^{\mathrm{op}}$, and checking that the maps agree is another straightforward computation.

Remark 5. This Lemma implies that the natural left module analogue of $\mathrm{AB}$, Lemma 5.4] holds (this may of course also be proved directly). References to [AB, Lemma 5.4] below will often implicitly be to its left module analogue.

\section{Canonical Dimensions for Iwasawa algebras}

Let us first fix some notation and terminology. As in the previous section we let $K$ be a finite field extension of $\mathbb{Q}_{p}$. In this section we let $G$ denote a compact $p$-adic analytic group. For the definition, see DdSMS99, §8]; note that for us a $p$-adic analytic group is the same as a $\mathbb{Q}_{p}$-analytic group. Put $d=\operatorname{dim} G$ (this is the dimension of $G$ as a $\mathbb{Q}_{p}$-analytic group, so e.g., $\left.\operatorname{dim} S L_{2}(K)=3\left[K: \mathbb{Q}_{p}\right]\right)$. As in DdSMS99, §9.5] we let $\mathcal{L}(G)$ denote the Lie algebra of $G$; this is a $\mathbb{Q}_{p}$-Lie algebra of dimension $d$. We let $K G$ denote the Iwasawa algebra of $G$ with coefficients in $K$, defined as $K G=\left(\lim _{\longleftarrow} \mathcal{O}_{K}[G / N]\right) \otimes_{\mathcal{O}_{K}} K$, where the inverse limit runs through all open normal subgroups $N \subseteq G$. $K G$ is Auslander-Gorenstein with self-injective dimension $d$ (see [AW1, Definition 2.5] for this notion). This follows for example from [AB, Lemma 5.4], [AW1, Lemma 10.13] and the existence of a uniform normal open subgroup of $G$ (see below for this notion). For any Auslander-Gorenstein ring $A$, we define the grade $j_{A}(M)$ and canonical dimension $d_{A}(M)$ of any non-zero finitely generated $A$-module $M$ by

$$
\begin{gathered}
j_{A}(M)=\min \left\{j \mid \operatorname{Ext}_{A}^{j}(M, A) \neq 0\right\}, \\
d_{A}(M)=\operatorname{inj} \operatorname{dim}_{A} A-j_{A}(M) .
\end{gathered}
$$

See [AW1, §2.5] for more definitions and details. We will also use the notion of a uniform pro-p group, for which we refer to DdSMS99, $\S 4$ and $\S 8.3$ ]. A uniform pro- $p$ group $H$ has an associated $\mathbb{Z}_{p}$-Lie algebra $L_{H}$ (see [DdSMS99, §4.5]) which is 
free as a $\mathbb{Z}_{p}$-module of rank $\operatorname{dim} H$. The remainder of this section will be devoted to the proof of Theorem 1. First, we record a simple reduction:

Lemma 6. It suffices to prove Theorem 1 for uniform pro-p groups and for $K=$ $\mathbb{Q}_{p}$.

Proof. Pick a uniform normal open subgroup $H$ of $G$. Let $M$ be a finitely generated $K G$-module. Then $\mathcal{L}(G)=\mathcal{L}(H)$, so $r_{G}=r_{H}$, and [AB, Lemma 5.4] implies that $d_{K G}(M)=d_{K H}(M)$. Hence we may reduce to uniform pro- $p$ groups. Then [AW1, Lemma 2.6] implies that it suffices to prove it for $K=\mathbb{Q}_{p}$.

Before we proceed we need to recall the rings $\mathcal{U}_{n} * H_{n}$, introduced in [AW1, §10.6]. From now on let us assume that $G$ is uniform and $K=\mathbb{Q}_{p}$. We may then set $\mathfrak{h}=p^{-1} L_{G}$; since $L_{G}$ satisfies $\left[L_{G}, L_{G}\right] \subseteq p L_{G}, \mathfrak{h}$ is a $\mathbb{Z}_{p}$-sub-Lie algebra of $\mathcal{L}(G)$, finite free of rank $d$. Recall that, for all $n \in \mathbb{Z}_{\geq 0}$, the groups $G^{p^{n}}$ are normal uniform pro- $p$ subgroups of $G$ with $L_{G^{p^{n}}}=p^{n} L_{G}$ and we set $H_{n}=G / G^{p^{n}}$. In AW1, $\S 10.6], \mathcal{U}_{n}$ is defined to be the microlocalisation of $\mathbb{Z}_{p} G^{p^{n}}$ with respect to the microlocal Ore set $S_{n}=\bigcup_{a>0} p^{a}+\mathfrak{m}_{n}^{a+1}$, where $\mathfrak{m}_{n}$ is the unique (left and right) maximal ideal of $\mathbb{Z}_{p} G^{p^{n}}$. If we need to emphasize the group $G$ we will write $\mathcal{U}_{n}(G)$. By [AW1, Theorem 10.4], $\mathcal{U}_{n}$ is isomorphic to $\widehat{U\left(p^{n} \mathfrak{h}\right)_{\mathbb{Q}_{p}}} \cdot \mathbb{Z}_{p} G$ is a crossed product $\mathbb{Z}_{p} G^{p^{n}} * H_{n}$ and since $S_{n}$ is invariant under automorphisms we obtain a canonical homomorphism $\operatorname{Aut}\left(\mathbb{Z}_{p} G^{p^{n}}\right) \rightarrow \operatorname{Aut}\left(\mathcal{U}_{n}\right)$. Note that if $f_{1}, f_{2} \in \operatorname{Aut}\left(\mathcal{U}_{n}\right)$ come from $\operatorname{Aut}\left(\mathbb{Z}_{p} G^{p^{n}}\right)$ and agree on $\mathbb{Z}_{p} G^{p^{n}}$ then they are equal (this is immediate from the construction). Thus we may form $\mathcal{U}_{n} * H_{n}$ by Lemma $4(2)$ and we get an induced homomorphism $\mathbb{Z}_{p} G \rightarrow \mathcal{U}_{n} * H_{n}$. Since $p$ is invertible in $\mathcal{U}_{n} * H_{n}$, we have a homomorphism $\mathbb{Q}_{p} G \rightarrow \mathcal{U}_{n} * H_{n}$. We will need some left/right complements to various results in $\mathrm{AW} 1$ :

Proposition 7. 1) The natural map $\mathbb{Q}_{p} G \rightarrow \mathcal{U}_{n} * H_{n}$ is left and right flat.

2) If $M$ is a finitely generated left $\mathbb{Q}_{p} G$-module, then $\left(\mathcal{U}_{n} * H_{n}\right) \otimes_{\mathbb{Q}_{p} G} M=0$ if and only if $M$ is $S_{n}$-torsion.

3) If $M$ is a finitely generated right $\mathbb{Q}_{p} G$-module, then $M \otimes_{\mathbb{Q}_{p} G}\left(\mathcal{U}_{n} * H_{n}\right)=0$ if and only if $M$ is $S_{n}$-torsion.

4) If $M$ is a finitely generated $p$-torsion free left or right $\mathbb{Z}_{p} G$-module, then there exists an $n_{0} \in \mathbb{Z}_{\geq 0}$ such that $M$ is $S_{n}$-torsion-free for all $n \geq n_{0}$.

Proof. 1) Right flatness is [AW1, Proposition 10.6(d)] and from this we also get right flatness of $\mathbb{Q}_{p} G^{\text {op }} \rightarrow \mathcal{U}_{n}\left(G^{\text {op }}\right) * H_{n}^{\text {op }}$ (i.e., performing the same constructions for the opposite group). However, we have identifications $\mathbb{Q}_{p} G^{\text {op }}=\left(\mathbb{Q}_{p} G\right)^{\text {op }}$ and $\mathcal{U}_{n}\left(G^{\text {op }}\right) *$ $H_{n}^{\text {op }}=\left(\mathcal{U}_{n}(G) * H_{n}\right)^{\text {op }}$ identifying $\mathbb{Q}_{p} G^{\text {op }} \rightarrow \mathcal{U}_{n}\left(G^{\text {op }}\right) * H_{n}^{\text {op }}$ with $\left(\mathbb{Q}_{p} G\right)^{\text {op }} \rightarrow$ $\left(\mathcal{U}_{n}(G) * H_{n}\right)^{\text {op }}$ using Lemma 4, so we get the desired left flatness.

2 ) is AW1, Proposition 10.6(e)] and 3) follows from 2) applied to $G^{\text {op }}$ with the identifications in the proof of 1). Similarly, the left part of 4) is [AW1, Corollary 10.11] and the right part follows as above.

Before we get to the proof of the main theorem we will abstract a short calculation from the proof: 
Lemma 8. Let $A, B, S$ and $T$ be rings and assume that we have a commutative diagram

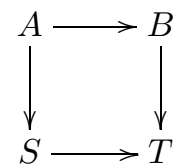

where $A \rightarrow S$ makes $S$ into a crossed product $A * G$ for some finite group $G$. Let $M$ be a finitely generated left $S$-module and assume that $\operatorname{Ext}_{S}^{i}(M, S) \otimes_{S} T \neq 0$ for some $i$. Then

$$
\operatorname{Ext}_{A}^{i}(M, A) \otimes_{A} B \neq 0 .
$$

Proof. Assume that $\operatorname{Ext}_{A}^{i}(M, A) \otimes_{A} B=0$. Then $\operatorname{Ext}_{A}^{i}(M, A) \otimes_{A} T=0$. However, by Remark $5 \operatorname{Ext}_{A}^{i}(M, A)=\operatorname{Ext}_{S}^{i}(M, S)$ as right $A$-modules. Thus $\operatorname{Ext}_{S}^{i}(M, S) \otimes_{A}$ $T=0$. But $\operatorname{Ext}_{S}^{i}(M, S) \otimes_{A} T$ surjects onto $\operatorname{Ext}_{S}^{i}(M, S) \otimes_{S} T$, a contradiction.

We now come to the proof of Theorem 1. Recall from AW1, Theorem 3.3, Proposition 9.1(b)] and the paragraph below it that $\mathcal{U}_{n}$ is Auslander-Gorenstein with self-injective dimension $d$ (in fact it is Auslander regular). By $\mathrm{AB}$, Lemma 5.4] $\mathcal{U}_{n} * H_{n}$ is also Auslander-Gorenstein of self-injective dimension $d\left(\mathcal{U}_{n} * H_{n}\right.$ is also Auslander regular). We will use this freely in the proof (in particular the fact that the self-injective dimensions agree).

Proposition 9. Theorem 1 is true when $G$ is uniform and $K=\mathbb{Q}_{p}$.

Proof. Let $M$ be a finitely generated $\mathbb{Q}_{p} G$-module which is not finite dimensional as a $\mathbb{Q}_{p}$-vector space. Let $j=j_{\mathbb{Q}_{p} G}(M)$ and set $N:=\operatorname{Ext}_{\mathbb{Q}_{p} G}^{j}\left(M, \mathbb{Q}_{p} G\right)$, this is a finitely generated right $\mathbb{Q}_{p} G$-module. By Proposition 7 we can find $t \geq 0$ such that $N$ is $S_{n^{-}}$-torsion-free for all $n \geq t$. Now $N=\operatorname{Ext}_{\mathbb{Q}_{p} G^{p^{n}}}^{j}\left(M, \mathbb{Q}_{p} G^{p^{n}}\right)$ and $d_{\mathbb{Q}_{p} G}(M)=d_{\mathbb{Q}_{p} G^{p^{n}}}(M)$ for any $n \geq 0$ by [AB, Lemma 5.4], so by replacing $G$ by $G^{p^{t}}$ if necessary we may assume $t=0$. Thus we can assume that $N$ is $S_{0}$-torsion-free.

Let $F$ be a finite extension of $\mathbb{Q}_{p}$ such that $\mathcal{L}(G) \otimes_{\mathbb{Q}_{p}} F$ is a split $F$-Lie algebra. Then we may find a split semisimple simply connected algebraic group $\mathbb{G} / \mathcal{O}_{F}$ whose $\mathcal{O}_{F}$-Lie algebra $\mathfrak{g}$ satisfies $\mathfrak{g} \otimes_{\mathcal{O}_{F}} F \cong \mathcal{L}(G) \otimes_{\mathbb{Q}_{p}} F$. We fix this isomorphism throughout and consider $\mathfrak{g}$ as a subset of $\mathfrak{g} \otimes_{\mathcal{O}_{F}} F$. Recall that $\mathfrak{h}=p^{-1} L_{G}$; we can find integers $n, m \geq 0$ such that

$$
p^{n}\left(\mathfrak{h} \otimes_{\mathbb{Z}_{p}} \mathcal{O}_{F}\right) \subseteq p^{m} \mathfrak{g} \subseteq \mathfrak{h} \otimes_{\mathbb{Z}_{p}} \mathcal{O}_{F} .
$$

Now because $N$ is $S_{0}$-torsion-free, $N \otimes_{\mathbb{Q}_{p} G} \mathcal{U}_{0}$ is non-zero by AW1, Proposition 10.6(e)], and therefore $N \otimes_{\mathbb{Q}_{p} G}\left(\mathcal{U}_{n} * H_{n}\right)$ is also non-zero since $\mathbb{Q}_{p} G \rightarrow \mathcal{U}_{0}$ factors through $\mathbb{Q}_{p} G \rightarrow \mathcal{U}_{n} * H_{n}$. By applying Proposition 7 together with [AW1, Proposition 2.6] we deduce that

$$
d_{\mathbb{Q}_{p} G}(M)=d_{\mathcal{U}_{0}}\left(M_{0}\right)=d_{\mathcal{U}_{n} * H_{n}}\left(M_{n}\right)
$$

where $M_{n}:=\left(\mathcal{U}_{n} * H_{n}\right) \otimes_{\mathbb{Q}_{p} G} M$. We may now again apply [AB, Lemma 5.4] to deduce that

$$
d_{\mathcal{U}_{n} * H_{n}}\left(M_{n}\right)=d_{\mathcal{U}_{n}}\left(M_{n}\right) .
$$

Furthermore, [AW1, Lemmas 2.6 and 3.9] give us that

$$
d_{\mathcal{U}_{n}}\left(M_{n}\right)=d_{F \otimes_{\mathbb{Q}_{p}} \mathcal{U}_{n}}\left(F \otimes_{\mathbb{Q}_{p}} M\right) \quad \text { and } \quad F \otimes_{\mathbb{Q}_{p}} \mathcal{U}_{n}=U\left(p^{n} \widehat{\mathfrak{h} \otimes_{\mathbb{Z}_{p}}} \mathcal{O}_{F}\right)_{F} .
$$


Since $F \otimes_{\mathbb{Q}_{p}} \mathcal{U}_{n} \rightarrow F \otimes_{\mathbb{Q}_{p}} \mathcal{U}_{0}$ factors through $\widehat{U\left(p^{m} \mathfrak{g}\right)_{F}}$, there is a commutative diagram

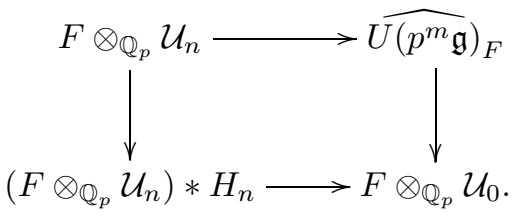

Let $S=\left(F \otimes_{\mathbb{Q}_{p}} \mathcal{U}_{n}\right) * H_{n}$ and $T=F \otimes_{\mathbb{Q}_{p}} \mathcal{U}_{0}$, and note that $F \otimes_{\mathbb{Q}_{p}} M_{n}=S \otimes_{\mathbb{Q}_{p} G} M$, so that

$$
\begin{aligned}
\operatorname{Ext}_{S}^{j}\left(F \otimes_{\mathbb{Q}_{p}} M_{n}, S\right) \otimes_{S} T & \cong \operatorname{Ext}_{S}^{j}\left(S \otimes_{\mathbb{Q}_{p} G} M, S\right) \otimes_{S} T \\
& \cong \operatorname{Ext}_{\mathbb{Q}_{p} G}^{j}\left(M, \mathbb{Q}_{p} G\right) \otimes_{\mathbb{Q}_{p} G} T=N \otimes_{\mathbb{Q}_{p} G} T \neq 0
\end{aligned}
$$

because $N \otimes_{\mathbb{Q}_{p} G} \mathcal{U}_{0} \neq 0$ by construction. Hence Lemma 8 implies that

$$
\operatorname{Ext}_{F \otimes_{\mathbb{Q}_{p}} \mathcal{U}_{n}}^{j}\left(F \otimes_{\mathbb{Q}_{p}} M_{n}, F \otimes_{\mathbb{Q}_{p}} \mathcal{U}_{n}\right) \otimes_{F \otimes_{\mathbb{Q}_{p}} \mathcal{U}_{n}} \widehat{U\left(p^{m} \mathfrak{g}\right)_{F}} \neq 0 .
$$

Since $p^{n} \mathfrak{h} \otimes_{\mathbb{Z}_{p}} \mathcal{O}_{F} \subseteq p^{m} \mathfrak{g}$ and $\left[p^{m} \mathfrak{g}, p^{n} \mathfrak{h} \otimes_{\mathbb{Z}_{p}} \mathcal{O}_{F}\right] \subseteq p^{n} \mathfrak{h} \otimes_{\mathbb{Z}_{p}} \mathcal{O}_{F}$, Lemma 3 implies that the top arrow in the above commutative diagram $F \otimes_{\mathbb{Q}_{p}} \mathcal{U}_{n} \rightarrow \widehat{U\left(p^{m} \mathfrak{g}\right)_{F}}$ is left and right flat. Thus [AW1, Proposition 2.6] applied to the top arrow in the commutative diagram above gives

$$
d_{\mathbb{Q}_{p} G}(M)=d_{F \otimes_{\mathbb{Q}_{p}} \mathcal{U}_{n}}\left(F \otimes_{\mathbb{Q}_{p}} M_{n}\right)=d_{\widehat{U\left(p^{m} \mathfrak{g}\right)_{F}}}(V)
$$

where $V:=\widehat{U\left(p^{m} \mathfrak{g}\right)_{F}} \otimes_{F \otimes_{\mathbb{Q}_{p}} \mathcal{U}_{n}}\left(F \otimes_{\mathbb{Q}_{p}} M_{n}\right)$. Here we have used the fact that $\widehat{U\left(p^{m} \mathfrak{g}\right)_{F}}$ is Auslander regular of self-injective dimension $d$. Now [AW1, Lemma 10.13] implies that $d_{\mathbb{Q}_{p} G}(M) \geq 1$ since $M$ is not finite dimensional as a $\mathbb{Q}_{p^{-}}$ vector space (we note here that [AW1, Lemma 10.13] does not require $G$ to satisfy the assumptions of [AW1, §10.12]). Finally, [AW1, Theorem 9.10] implies that $d_{\widehat{U\left(p^{m} \mathfrak{g}\right)_{F}}}(V) \geq r$, so $d_{\mathbb{Q}_{p} G}(M) \geq r$ as desired.

\section{Canonical dimensions for Distribution algebras}

In this section we let $L \subseteq K$ be finite extensions of $\mathbb{Q}_{p}$ and let $G$ be a locally $L$ analytic group. We let $D(G, K)$ denote the algebra of $K$-valued distributions on $G$, studied in depth in [ST2], which we refer to for more details and some terminology.

Following the notation of $\left[\mathrm{ST2}\right.$ we let $G_{0}$ denote the underlying $\mathbb{Q}_{p}$-analytic group obtained from $G$ by forgetting the $L$-structure. Recall (see e.g. [Sch2, §5.6]) that $G$ is said to be $L$-uniform if $G_{0}$ is uniform and $L_{G_{0}} \subseteq \mathcal{L}(G)$ is an $\mathcal{O}_{L}$-lattice. If this holds we will write $L_{G}$ for $L_{G_{0}}$ with its $\mathcal{O}_{L}$-module structure. When $G$ is $L$-uniform, $D(G, K)$ carries a Fréchet-Stein structure given by the inverse system $\left(D_{r}(G, K)\right)_{r \in\left[p^{-1}, 1\right) \cap p^{\mathbb{Q}}}$ (see e.g. [Sch2, $\left.\left.\S 5.17\right]\right)$. We put $r_{n}=p^{-1 / p^{n}}$ and will from now on only consider the inverse system $\left(D_{r_{n}}(G, K)\right)_{n \in \mathbb{Z}_{>0}}$, which is final in the previous inverse system. The rings $D_{r_{n}}(G, K)$ are Auslander regular of self-injective and global dimension $d=\operatorname{dim}_{L} G$ when $n \geq 1$ by Sch2, Proposition 9.3]. Thus, the category of coadmissible $D(G, K)$-modules ([ST2, §6]) has a well-defined dimension theory ([ST2, §8]). Note that if $G$ is $L$-uniform then so is $G^{p^{n}}$ for all $n \geq 0$, and we have $D_{r_{0}}\left(G^{p^{n}}, K\right) * H_{n}=D_{r_{n}}(G, K)$ by [Sch2, Corollary 5.13] with $H_{n}=G / G^{p^{n}}$ as in the previous section. 
When $G$ is a general locally $L$-analytic group, $G$ has at least one (and hence many) open $L$-uniform subgroup(s); indeed, any compact open locally $L$-analytic subgroup of $G$ has a basis of neighbourhoods of the identity consisting of open normal $L$-uniform subgroups by Sch1, Corollary 4.4]. Thus the category of coadmissible $D(G, K)$-modules has a well-defined dimension theory using the formalism of [ST2, §8], defined by restriction to an arbitrary open $L$-uniform subgroup. Therefore it suffices, by definition, to prove Theorem 2 for $L$-uniform groups.

We recall the link between affinoid enveloping algebras and distribution algebras. The first part of the following version of the Lazard isomorphism is essentially proved in [Sch2, §6.6] but stated only in a special case; it was then proven (in somewhat more generality) in [AW2, Lemma 5.2]. We give a brief sketch of the proof for the convenience of the reader.

Proposition 10. Assume that $G$ is L-uniform. Then there is an isomorphism $\Psi_{G}: \widehat{U(\mathfrak{g})}_{K} \rightarrow D_{r_{0}}(G, K)$, where $\mathfrak{g}=p^{-1} L_{G}$. It is compatible with morphisms $\alpha: G \rightarrow H$ in the sense that the diagram

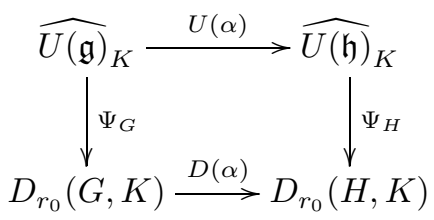

commutes where $U(\alpha)$ and $D(\alpha)$ are the natural maps induced by $\alpha$.

Proof. We let $\mathfrak{g}_{0}$ denote $\mathfrak{g}$ thought of as a $\mathbb{Z}_{p^{-}}$Lie algebra by forgetting the $\mathcal{O}_{L^{-}}$ structure. Recall that there is a natural morphism $\mathcal{L}\left(G_{0}\right) \rightarrow D_{r_{0}}\left(G_{0}, K\right)$ factoring through $D\left(G_{0}, K\right)$ defined by

$$
X f=\left.\frac{d}{d t}(f(\exp (-t X)))\right|_{t=0}
$$

for $f \in \mathcal{C}^{\text {la }}\left(G_{0}, K\right)$ and $X \in \mathcal{L}\left(G_{0}\right)$ (see e.g. [Sch2, §5.2]); it gives an inclusion $\mathfrak{g} \rightarrow D_{r_{0}}(G, K)$. For example, by [Sch2, Proposition 6.3] there is an isomorphism

$$
\Psi_{G_{0}}:{\widehat{U\left(\mathfrak{g}_{0}\right)}}_{K}=U\left({\mathfrak{\mathfrak { g } _ { 0 }}}_{\otimes_{\mathbb{Z}_{p}}}^{\mathcal{O}_{L}}\right)_{K} \rightarrow D_{r_{0}}\left(G_{0}, K\right)
$$

which is compatible with the embeddings of $\mathfrak{g}_{0}$ on both sides. Put $\mathfrak{k}=\operatorname{ker}\left(\mathfrak{g}_{0} \otimes_{\mathbb{Z}_{p}}\right.$ $\left.\mathcal{O}_{L} \rightarrow \mathfrak{g}\right)$ where the map is given by $a \otimes X \mapsto a X$. Then $D_{r_{0}}(G, K)$ is the quotient of $D_{r_{0}}\left(G_{0}, K\right)$ by the ideal generated by $\mathfrak{k}$ by [Sch1, Lemma 5.1]. Similarly, $\widehat{U(\mathfrak{g})}{ }_{K}$

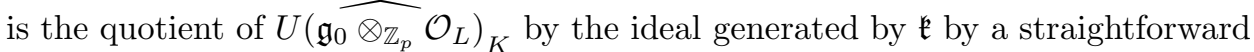
argument; using [Bour, §I.2.3, Proposition 3] we see that

$$
0 \rightarrow \mathfrak{k} . U\left(\mathfrak{g}_{0} \otimes_{\mathbb{Z}_{p}} \mathcal{O}_{L}\right) \rightarrow U\left(\mathfrak{g}_{0} \otimes_{\mathbb{Z}_{p}} \mathcal{O}_{L}\right) \rightarrow U(\mathfrak{g}) \rightarrow 0
$$

is an exact sequence of finitely generated $U\left(\mathfrak{g}_{0} \otimes_{\mathbb{Z}_{p}} \mathcal{O}_{L}\right)$-modules. Now use the Artin-Rees Lemma and tensor with $K$ to conclude. Thus $\Psi_{G_{0}}$ induces the desired isomorphism $\Psi_{G}$ by quotienting out by the ideal generated by $\mathfrak{k}$ on the source and target. Finally, the compatibility with morphisms follows from the functoriality of the morphism $\mathcal{L}\left(G_{0}\right) \rightarrow D_{r_{0}}\left(G_{0}, K\right)$, which is straightforward to check from the defining formula.

With these preparations we may now prove Theorem 2 (which, we recall, one only needs to show for $L$-uniform groups). 
Proposition 11. Theorem 2 holds for L-uniform groups $G$.

Proof. The proof is very similar to that of Proposition 9, First, note that without loss of generality $L=K$ by [AW1, Lemma 2.6]. For the purposes of this proof we put $D:=D(G, L)$ and $D_{n}:=D_{r_{n}}(G, L)$. Let $M$ be a coadmissible left $D$-module such that $d_{D}(M) \neq 0$, and put $N=\operatorname{Ext}_{D}^{j}(M, D)$ with $j=j_{D}(M)$. By [ST2, Lemma 8.4] we may find an integer $t \geq 0$ such that $N_{n}:=N \otimes_{D} D_{n}=\operatorname{Ext}_{D_{n}}\left(M_{n}, D_{n}\right) \neq 0$ for all $n \geq t$, where $M_{n}:=D_{n} \otimes_{D} M$. Because of the bimodule isomorphisms $D_{n} \cong D_{r_{0}}\left(G^{p^{n}}, L\right) \otimes_{D\left(G^{p^{n}}, L\right)} D$ and $D_{n} \cong D \otimes_{D\left(G^{p^{n}}, L\right)} D_{r_{0}}\left(G^{p^{n}}, L\right)$, we see that $M_{n} \cong D_{r_{0}}\left(G^{p^{n}}, L\right) \otimes_{D\left(G^{p^{n}}, L\right)} M$ and $N_{n} \cong N \otimes_{D\left(G^{p^{n}}, L\right)} D_{r_{0}}\left(G^{p^{n}}, L\right)$ for any $n \geq 0$. Therefore we may, as in the proof of Proposition 9. replace $G$ by $G^{p^{t}}$ and without loss of generality assume that $t=0$.

Pick a finite extension $F / L$ such that $\mathcal{L}(G) \otimes_{L} F$ is split and let $\mathfrak{g} \subseteq \mathcal{L}(G) \otimes_{L} F$ be a split $\mathcal{O}_{F}$-sub-Lie algebra. Write $\mathfrak{h}:=p^{-1} L_{G}$ and pick positive integers $m$ and $n$ such that

$$
p^{n} \mathfrak{h} \otimes_{\mathcal{O}_{L}} \mathcal{O}_{F} \subseteq p^{m} \mathfrak{g} \subseteq \mathfrak{h} \otimes_{\mathcal{O}_{L}} \mathcal{O}_{F}
$$

We have that $d_{D}(M)=d_{D_{n}}\left(M_{n}\right)$ and from here on we argue exactly as in the proof of Proposition 9, using that $D_{n}=D_{r_{0}}\left(G^{p^{n}}, L\right) * G / G^{p^{n}}=\widehat{U\left(p^{n} \mathfrak{h}\right)_{L}} * G / G^{p^{n}}$ using Proposition 10. Note that the compatibility statement in Proposition 10 ensures that the diagram that Lemma 8 has to be applied to, namely

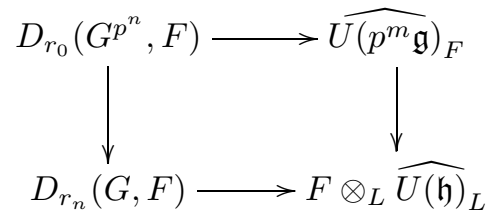

is commutative.

\section{ACKNOWLEDGEMENTS}

The second author was supported by National Science Foundation grants 093207800 and DMS-1128155 during the work on this paper whilst a member of MSRI and the IAS, respectively, and would like to thank both institutions for their hospitality. The first author was supported by EPSRC grant EP/L005190/1. Both authors are grateful to the referee for a careful reading of this paper.

\section{REFERENCES}

[AB] K. Ardakov and K. A. Brown, Primeness, semiprimeness and localisation in Iwasawa algebras, Trans. Amer. Math. Soc. 359 (2007), no. 4, 1499-1515, DOI 10.1090/S00029947-06-04153-5. MR.2272136(2007j:16031)

[AW1] K. Ardakov and S. Wadsley, On irreducible representations of compact p-adic analytic groups, Ann. of Math. (2) 178 (2013), no. 2, 453-557, DOI 10.4007/annals.2013.178.2.3. MR3071505

[AW2] K. Ardakov and S. Wadsley, Verma modules for Iwasawa algebras are faithful, Münster J. Math. 7 (2014), no. 1, 5-26. MR3271237

[Bour] N. Bourbaki, Groupes et algébres de Lie, Chapitre 1, Hermann 2007.

[DdSMS99] J. D. Dixon, M. P. F. du Sautoy, A. Mann, and D. Segal, Analytic pro-p groups, 2nd ed., Cambridge Studies in Advanced Mathematics, vol. 61, Cambridge University Press, Cambridge, 1999. MR1720368 (2000m:20039) 
[Em] M. Emerton, Locally analytic vectors in representations of locally $p$-adic analytic groups. http://www.math.uchicago.edu/ emerton/pdffiles/analytic.pdf. To appear in Memoirs of the AMS.

[Pa] D. S. Passman, Infinite crossed products, Pure and Applied Mathematics, vol. 135, Academic Press, Inc., Boston, MA, 1989. MR979094 (90g:16002)

[Sch1] T. Schmidt, Auslander regularity of $p$-adic distribution algebras, Represent. Theory 12 (2008), 37-57, DOI 10.1090/S1088-4165-08-00323-3. MR2375595(2009b:22018)

[Sch2] T. Schmidt, On locally analytic Beilinson-Bernstein localization and the canonical dimension, Math. Z. 275 (2013), no. 3-4, 793-833, DOI 10.1007/s00209-013-1161-x. MR.3127038

[SS] T. Schmidt, M. Strauch, Dimensions of some locally analytic representations, Represent. Theory 20 (2016), 14-38. DOI: http://dx.doi.org/10.1090/ert/475.

[ST1] P. Schneider and J. Teitelbaum, Banach space representations and Iwasawa theory, Israel J. Math. 127 (2002), 359-380, DOI 10.1007/BF02784538. MR.1900706 (2003c:22026)

[ST2] P. Schneider and J. Teitelbaum, Algebras of p-adic distributions and admissible representations, Invent. Math. 153 (2003), no. 1, 145-196, DOI 10.1007/s00222-002-0284-1. MR:1990669 (2004g:22015)

School of Mathematical Sciences, University of Nottingham, University Park, NotTINGHAM NG7 2RD, United KingDOM

E-mail address: ardakov@maths.ox.ac.uk

School of Mathematics, Institute for Advanced Study, Einstein Drive, Princeton, NEW JERSEY 08540

E-mail address: johansson@math.ias.edu 